\title{
Ein Gegenvorschlag als Ausweg aus der Sackgasse
}

Zurzeit behandelt das Parlament die Initiative «Für eine soziale Einheitskasse». Zwischen 1996 und 2004 sind die Krankenkassenprämien um $62 \%$ gestiegen, und dieser Trend hat sich seither fortgesetzt. Die Massnahmen zur Eindämmung dieser Entwicklung hatten bisher vor allem restriktiven und autoritären Charakter, zielten jedoch nie auf die grundlegenden Probleme ab.

Die Initiative des «Mouvement Populaire des Familles» will auf diese Situation reagieren. Die neue Einheitskasse soll nach paritätischem Modell mit Vertretung der staatlichen Instanzen, der Leistungserbringer und der Versicherten im Aufsichtsrat funktionieren. Ausserdem müssten die Prämien einkommensabhängig bezahlt werden.

Damit würde das aktuelle System völlig umgekrempelt. Eine solch grundlegende Änderung stösst jedoch unmittelbar auf zahlreiche nur schwer zu überwindende Hindernisse: Die Liquidation der bestehenden Krankenkassen, die Verteilung ihres Vermögens und die Übertragung ihrer Kundenstämme auf die neue Einheitskasse würden ein riesiges Feld für Konflikte eröffnen, für die keine schnelle Lösung in Sicht ist. Müssen wir wirklich so weit gehen, um die Konfrontation zu beenden und die aktuellen Missstände zu beheben?

Der Zentralvorstand der FMH ist überzeugt, dass das Schweizer Gesundheitswesen mit etwas gesundem Menschenverstand und Pragmatismus wieder in ordentliche Bahnen gelenkt werden kann, ohne zu derart extremen Massnahmen zu greifen.

Die sozioökonomischen Schwierigkeiten und die Probleme aufgrund der demographischen Entwicklung oder des medizinischen Fortschritts können nur unter Wahrung unserer Grundwerte, insbesondere der persönlichen Beziehung zum Patienten, sowie durch Verständigung unter Partnern und Einbringung der jeweiligen Kompetenzen gelöst werden.

In diesem Sinne hat die FMH bei der nationalrätlichen Kommission für soziale Sicherheit und Gesundheit einen direkten Gegenvorschlag zu dieser Initiative in Form von drei Absätzen zur Ergänzung von Artikel 117 der Bundesverfassung eingereicht. Sie finden diesen Gegenvorschlag auf Seite 295 dieser Ausgabe der SÄZ. Nachfolgend einige Bemerkungen dazu.
Partnerschaft bedeutet auch Demokratie und Repräsentativität. Die Krankenkassen haben offensichtlich den partnerschaftlichen Geist verloren, der ihrer Schaffung zugrunde lag. Ihre hauptsächlich aufgrund persönlicher, politischer oder wirtschaftlicher Beziehungen besetzten Führungsorgane vertreten letztendlich nur sich selbst und haben keinen Bezug zur Basis. Ihre Hauptaufgabe wäre jedoch, dank einer demokratischen und partizipativen Struktur ihren Mitgliedern und deren Interessen zu dienen.

Die Tätigkeiten im Rahmen der obligatorischen sozialen Krankenversicherung und in dem der Zusatzversicherungen oder der übrigen privaten Versicherungen verfolgen nicht die gleichen strategischen Ziele. Daraus entstehen offensichtliche und schädliche Interessenkonflikte, welche die Atmosphäre vergiften und klar zu Lasten der sozialen Krankenversicherung gehen. Mit einer strukturellen, organisatorischen und wirtschaftlichen Trennung dieser beiden Sektoren (soziale und private Krankenversicherung) können die Dinge wieder zurechtgerückt und die jeweiligen Rollen geklärt werden, wovon alle Beteiligten profitieren werden.

Kurz gesagt: Die bis jetzt abwartende und zögerliche Haltung sorgt für zunehmende Verärgerung. Aus diesem Grund kann man die Initiative «Für eine soziale Einheitskasse» nicht einfach vom Tisch wischen, wie es der Bundesrat vorhat, und weiter nichts tun oder sich in einer strittigen und umstrittenen KVG-Revision verlieren.

Der Zentralvorstand der FMH hat eine ausgewogene und realistische Lösung vorgeschlagen, die bei einer Volksabstimmung von einer Mehrheit unterstützt werden kann. Der Gegenvorschlag hat bisher im Parlament angesichts von Partikularinteressen und politischen Ränkespielen keine Gnade gefunden. Wir wollen uns jedoch weiter dafür einsetzen, dass er zum Erfolg geführt wird.

Jacques de Haller, Präsident der FMH

Yves Guisan, Vizepräsident der FMH, Nationalrat 